\title{
Development of a Novel Wearable Ring-Shaped Biosensor*
}

\author{
L. Santarelli, O. Diyakonova, S. Betti, D. Esposito, E. Castro and F. Cavallo, Member, IEEE
}

\begin{abstract}
We report on the preliminary results obtained out of a wearable module designed to be encompassed within a ring-shaped system aimed at providing healthcare services. The module is composed of two sensors for the measuring of Galvanic Skin Response (GSR) and Heart Rate Variability (HRV). A first device validation was carried out by involving four subjects who were asked to perform tasks providing different stress-related statuses. A comparison of physiological parameters measured by the module with those measured by a commercial HRV-GSR sensor chosen as gold standard was made. Two out of the three HRV parameters and all of the GSR parameters measured with the module resulted consistent (mostly differing less than $10 \%$ ) with the same parameters measured by the gold standard. The work reported in this paper set a milestone for the realization of a system exploiting sensor fusion to provide active ageing, stress detection, activity recognition and e-health services has been achieved.
\end{abstract}

\section{INTRODUCTION}

Off-the-shelf Wearable Technology (WT) is progressively expanding and research institutes and companies are more and more prone to invest in such technology, in particular for applications in the fields of healthcare services and fitness [1]-[4]. WT functionalities are provided by using inertial sensors, aimed at the body motion tracking, and by means of Physiological Signal Measuring Sensors (PSMS), such as those aimed at the monitoring of the Heart Rate Variability (HRV) and Galvanic Skin Response (GSR) [5]. GSR activity is linked to sympathetic arousal, induced by external stimuli, causing peripheral sweating altering the positive/negative balance of ions hence resulting in an increase/decrease of the skin resistance [6]. GSR is particularly suited to be measured over palms or fingers since such districts are rich of sweat glands [7]. HRV is measured out of the identification of intervals variation between consecutive $\mathrm{R}$ peaks (QRS complex) by means of either optoelectronic devices, mostly located over wrist or fingers [8], [9], or MEMS sensing body vibration related to cardiac activity [10], [11].

Unobtrusiveness and easy-wearability are crucial to PSMS since cumbersome systems can affect body activity and cause artefacts, whilst lack of adhesion between PSMS probe and body surface yields poor Signal-to-Noise Ratio (SNR) or unintelligible signals [12]. Examples of commercial WT systems conceived addressing such criticalities are: Empatica E4 wristband or the Shimmer3 GSR+, both systems able to monitor HRV and GSR parameters at once; Zephyr ${ }^{\mathrm{TM}}$ BioHarness 3.0, a chest-belt monitoring both the body motion

*This work is supported by DAPHNE project (REGIONE TOSCANA PAR FAS 2007-2013, BANDO FAS SALUTE 2014, CUP J52I16000170002).

L. Santarelli, O. Diyakonova, D. Esposito, S. Betti., E.Castro and F. Cavallo* are with The BioRobotics Institute, Scuola Superiore Sant'Anna, Pisa, Italy (corresponding author e-mail: f.cavallo@ sssup.it) and the HRV; the ring shaped HRV monitoring system Motiv. Despite the progress made by these systems, especially in terms of wearability (Motiv) and multifunctionality (Shimmer3 GSR+, Empatica E4), WT specifically offering complex services for healthcare, such as early-diagnosis for mental degenerative diseases or long-term monitoring of workers activity in order to prevent professional diseases or stress-related risky situations, are still missing or only in the early stages of development.

Interestingly, we developed a WT solution named DAPHNE [2], [3], [13]-[15] based on inertial sensors that provides services for the early diagnosis of Parkinson's Disease (PD) and at home health-care assistance. Such system is CE certified and it is currently undergoing an experimentation stage in hospitals.

In this work, we report on the design and the realization of a novel module aimed at monitoring both GSR and HRV to be integrated within the ring-shaped DAPHNE system. A first prototype of the module was obtained and tested on four different subjects who worn the prototype during three different stages: a stimuli-free stage (Relax), a mental stress inducing stage (Mental Stress); a motor stress inducing stage (Physical Stress). Subjects were also wearing a Shimer3GSR+, acting as gold standard, in parallel to the module prototype. Therefore, a set of HRV and GSR parameters was obtained from each of the two systems out of all subjects involved. The test shed light on prototype effectiveness in detecting physiological response to different external stimuli. The integration of the module within DAPHNE system will empower the latter of long-term activity monitoring and gesture recognition as well as of precision medicine services.

\section{MATERIAL AND MethodS}

\section{A. System Circuit}

The module was developed by using a STM32-F401 Nucleo Board (STMicroelectronics), which is based on an ARM Cortex 3 processor. Such processor is the same on board the DAPHNE system, therefore an easy-to-plug approach was followed by mimicking the same conditions the PSMS will find once embedded on board the DAPHNE system. The PSMS chosen are: a MAXIM 30100 sensor for the detection of HRV, which is controlled by means of a I2C port; a SeedGrove GSR sensor, connected to a DC input port. Communication between the module and computer is enabled by a SPBT2632C2A class-2 Bluetooth module (STMicroelectronics) communicating to a SPBT3ATV3 dongle (STMicroelectronics). Data acquired were processed by using a homemade interface based on $\mathrm{C \#}$ language developed in Visual Studio 2015 (Microsoft Corporation). 


\section{B. HRV and GSR Signal Acquisition and Analysis}

The frequency sampling of the module prototype is $100 \mathrm{~Hz}$ for both the HRV and the GSR signal, whilst the gold standard features a sampling frequency of $51.2 \mathrm{~Hz}$. Both the HRV signal acquired by the module prototype and the Shimmer3 GSR+ were processed by means of the HRVAS free software [16]. The HRV parameters extracted by using this software are [11]:

- HR Mean, i.e. mean value of the Hearth Rate;

- IBI Mean, i.e. mean of Inter-Beat Interval corresponding to R-to-R interval;

- SDNN, i.e. Standard Deviation of all Normal RR intervals ( $\mathrm{NN}$ intervals).

The GSR parameters are extracted by analysing time and amplitude features of so-called startles, or rather peaks elapsing between 1 and 5 seconds arising within the phasic activity of the skin resistance, which is extracted from the tonic signal by means of a moving average filter (moving window set to 5 seconds) [17]. The GSR signal acquired by both the module prototype and the gold standard were analysed by means of an ad-hoc made algorithm of startle detection and features extraction, developed in Matlab R2016a (MathWorks) [11], [18], [19]. The parameters extracted by such algorithm are:

- \#Startle, i.e. the number of Startle detected;

- Startle Amp, i.e. the mean and the standard deviation of a startle amplitude;

- Startle Time, i.e. the mean and standard deviation of a startle duration.

\section{Administered Test}

In order to evaluate the device performances, tests on four subjects were carried out (two male and two female), with age ranging between 30 and 34 years (mean age: 31.75 years, standard deviation: 1.78 years). An informative consent form was signed before starting the experimentation by each of the subjects, who also were asked to fill a questionnaire about the stress perceived during each of the tests they went through at the end of the whole experimentation. The Institution's Ethical Review Board approved all experimental procedures involving human subjects. Attention was paid on creating a proper device demonstration environment, therefore on providing different stages characterized by different stimuli for subject involved, expectably inducing different physiological reaction. Furthermore, the tests also aimed at providing stressing condition for the PSMS probe-body portion coupling, electrodes and wiring connections. The tests were organized according to the following sequence:

- A Relax phase, i.e. 5 minutes of stimuli free stage;

- A Mental stress phase, i.e. 5 minutes of mental stress-inducing tests;

- A Physical Stress, i.e. 5 minutes of physical stress-inducing tests.
In particular, during the Relax phase subjects were asked to remain sitting with closed eyes. No interaction between subjects and environment took place. Immediately after this phase a cognitive stress-inducing phase started by using the Arithmetical Reasoning, subtest of the Wechsler Adult Intelligence Scale-Fourth Edition (WAIS-IV), and part of the Paced Auditory Serial Addition Test (PASAT) [20], [21]. These tests involve verbal working memory by asking subjects to perform rapid mental calculation and to solve arithmetical problems or by asking to add the last two numbers listened from an auditory track each time a new number is presented (a sequence of numbers is presented; each time the subject hear a new number, he had to add it to the previous number). Due to the mental working load and the concentration required, mental stress is induced. All the trials occurred in a room with temperature and light conditions maintained constant for the entire duration.

\section{System and Gold Standard positioning}

The prototype was worn by subject on the left hand by positioning the Maxim 30100 on the index finger, while the two electrodes of the SeedGrove sensor on the medium finger and the annular finger. The Shimmer3+ GSR was located on the right wrist of subjects with its HRV sensor positioned on the index finger and its two GSR electrodes on the medium finger and the annular finger of the same hand (Figure 1).

Figure 1. The module prototype and the gold standard worn during the test by participants (above) and the expected integration to be achieved once the module is embedded into DAPHNE system (below).

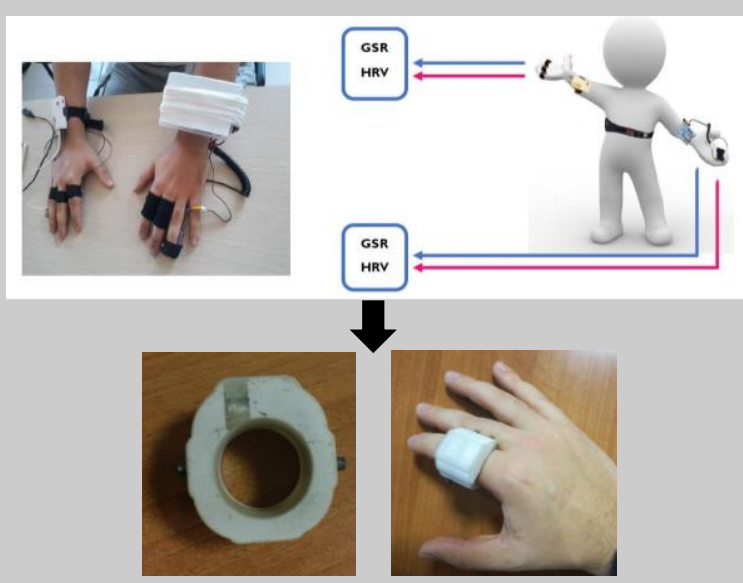

III. RESULTS

The GSR and HRV parameters measured during the Relax, the Mental stress and the Physical stress with both the gold standard and the module prototype are reported in TABLE I. Considering the Relax phase and the following Mental stress phase, both measuring systems yield HR mean and IBI mean values that follow an analogous trend for all subjects involved. Namely, the HRV increases passing from the Relax phase to the Mental Stress while the IBI decreases. A further increase of the HRV mean and decrease of the IBI mean take place passing from the Mental Stress to the Physical stress. The \#Startle also follows a coherent trend in all subjects and for both measuring systems, significantly increasing passing from the Relax Phase to the Mental stress phase, while decreasing during the physical stress with respect to the mental stress but remaining by far higher than the values registered in the relax phase. 
TABLE I.

FEATURES EXTRACTED FROM HRV AND GSR SIGNALS DURING EACH PHASE OF THE EXPERIMENTATION

\begin{tabular}{|c|c|c|c|c|c|c|c|c|c|}
\hline \multirow{2}{*}{\multicolumn{2}{|c|}{$\begin{array}{c}\text { Subjects } \\
\text { Device }\end{array}$}} & \multicolumn{2}{|c|}{ Subject 1} & \multicolumn{2}{|c|}{ Subjects 2} & \multicolumn{2}{|c|}{ Subject 3} & \multicolumn{2}{|c|}{ Subject 4} \\
\hline & & Prototype & Shimmer & Prototype & Shimmer & Prototype & Shimmer & Prototype & Shimmer \\
\hline \multirow{6}{*}{ Relax } & HR mean (bpm) & 58 & 57.6 & 75.8 & 74.2 & 60.6 & 60.6 & 61.6 & 61.4 \\
\hline & IBI Mean (ms) & 1035.136 & 1042.324 & 791.452 & 809.089 & 990.405 & 992.783 & 974.721 & 977.986 \\
\hline & $S D N N(m s)$ & 108.067 & 109.789 & 77.874 & 130.633 & 87.929 & 96.906 & 60.480 & 78.542 \\
\hline & \#Startle & 4 & 5 & 8 & 7 & 13 & 12 & 53 & 61 \\
\hline & Startle Time (s) & $2.255 \pm 0.545$ & $2.564 \pm 0.520$ & $2.177 \pm 0.856$ & $2.106 \pm 0.578$ & $2.601 \pm 0.668$ & $2.627 \pm 0.885$ & $1.867 \pm 0.569$ & $2.108 \pm$ \\
\hline & Startle Amp $(\mu S)$ & $0.056 \pm 0.055$ & $0.075 \pm 0.065$ & $0.065 \pm 0.056$ & $0.074 \pm 0.077$ & $0.021 \pm 0.006$ & $0.016 \pm 0.004$ & $0.108 \pm 0.077$ & $0.035 \pm 0.019$ \\
\hline \multirow{6}{*}{$\begin{array}{l}\text { Mental } \\
\text { Stress }\end{array}$} & HR mean (bpm) & 75.4 & 75.4 & 91.8 & 92 & 71 & 73.8 & 70 & 70 \\
\hline & IBI Mean (ms) & 797.281 & 796.668 & 654.806 & 651.882 & 843.661 & 813.469 & 858.341 & 858.551 \\
\hline & $S D N N(m s)$ & 70.529 & 75.546 & 106.472 & 91.051 & 106.634 & 148.212 & 82.783 & 79.196 \\
\hline & \#Startle & 77 & 79 & 77 & 75 & 59 & 77 & 112 & 100 \\
\hline & Startle Time (s) & $2.501 \pm 0.975$ & $2.814 \pm 0.952$ & $2.235 \pm 0.946$ & $2.214 \pm 0.936$ & $3.212 \pm 1.222$ & $2.938 \pm 1.153$ & $2.053 \pm 0.709$ & $1.925 \pm 0.656$ \\
\hline & Startle Amp $(\mu S)$ & $0.076 \pm 0.041$ & $0.106 \pm 0.058$ & $0.118 \pm 0.102$ & $0.068 \pm 0.037$ & $0.057 \pm 0.122$ & $0.035 \pm 0.026$ & $0.035 \pm 0.016$ & $0.033 \pm 0.013$ \\
\hline \multirow{6}{*}{$\begin{array}{l}\text { Physical } \\
\text { Stress }\end{array}$} & HR mean (bpm) & 103 & 102.8 & 120.4 & 118.4 & 101.6 & 100.6 & 104.8 & 111.4 \\
\hline & IBI Mean (ms) & 583.688 & 585.664 & 576.264 & 465.649 & 607.152 & 610.966 & 573.781 & 540.937 \\
\hline & SDNN (ms) & 81.869 & 69.013 & 728.382 & 83.921 & 107.782 & 95.739 & 342.898 & 60.279 \\
\hline & \#Startle & 54 & 59 & 95 & 98 & 47 & 57 & 55 & 64 \\
\hline & Startle Time (s) & $2.868 \pm 1.131$ & $2.278 \pm 0.974$ & $2.246 \pm 0.764$ & $1.745 \pm 0.462$ & $2.836 \pm 1.103$ & $2.679 \pm 1.116$ & $2.362 \pm 0.827$ & $2.181 \pm 0.767$ \\
\hline & Startle Amp $(\mu S)$ & $0.051 \pm 0.025$ & $0.112 \pm 0.058$ & $0.104 \pm 0.066$ & $0.068 \pm 0.053$ & $0.039 \pm 0.019$ & $0.073 \pm 0.031$ & $0.042 \pm 0.021$ & $0.035 \pm 0.016$ \\
\hline
\end{tabular}

TABLE II. ABSOLUTE MEAN PERCENTAGE VARIATION BETWEEN VALUES MEASURED WITH PROTOTYPE AND GOLD STANDARD SHIMMER3 GSR+

\begin{tabular}{|c|c|c|c|}
\hline \multicolumn{3}{|c|}{ Absolute Mean Percentage Variation against Gold Standard } \\
\hline Phases & Relax & Mental Stress & $\begin{array}{c}\text { Physical } \\
\text { Stress }\end{array}$ \\
\hline HR mean & $0.8 \%$ & $1 \%$ & $2.2 \%$ \\
\hline IBI Mean & $0.9 \%$ & $1.1 \%$ & $7.7 \%$ \\
\hline SDNN & $18.5 \%$ & $14 \%$ & $317 \%$ \\
\hline \#Startle & $13.9 \%$ & $10.1 \%$ & $10.8 \%$ \\
\hline Strartle Time & $6.9 \%$ & $7 \%$ & $17.2 \%$ \\
\hline Startle Amp & $69.2 \%$ & $43.6 \%$ & $43.4 \%$ \\
\hline
\end{tabular}

There is not a particular trend in the Startle Time and Startle Amp parameters, as expected being such parameters tendentiously taking place according the same dynamic in terms of time duration and signal amplitude. Nevertheless, there is a significant difference between the Startle Amp measured with the module prototype with respect to the one measured with the gold standard (up to $70 \%$ ). The SDNN parameter measured during the Mental stress and the Physical stress is not following coherent trends in the two systems inasmuch greatly increases according the prototype, whilst reduces according the golden standard. TABLE II. reports on the absolute mean percentage variation of parameters $(\Delta \%)$ measured with the prototype with respect to those measured by the gold standard. Interestingly, $\Delta \%$ between the HR mean and the IBI mean is within $1.1 \%$ for the relax phase and the mental phase, whilst it increases in the physical stress phase, in particular for the IBI resulting as great as $7.7 \%$. SDNN $\Delta \%$ is within the $20 \%$ in the Mental and Relax phases but it is over the $300 \%$ in the physical stress. The \#Startle and the Startle time $\Delta \%$ are within the $19 \%$ in all cases, whilst a significant difference is registered for the Startle Amp, with a $\Delta \%$ reaching nearly $70 \%$ in the Relax Phase. The stress effectively perceived during this phase was evaluated by subjects on average 6.5 out of 10 , according the questionnaires. The physical stress consisted in climbing up and down stairs for 5 minutes without any pause. The stress perceived during this test by participants was on average 5 out of 10 .

\section{DISCUSSION}

SDNN within the physical test presented the greatest criticalities, due to the unreasonable increase of such parameter and its inability to behave coherently with the gold standard. The increase of the standard deviation of R-R inter-peaks intervals is due to the decrease of the signal to noise ratio (SNR), in turn caused by a not optimized coupling of the HRV probe with the skin surface in the prototype, so allowing external light to reach the photodetector embedded within the MAXIM 30100 sensor. In fact, the Shimmer golden standard HRV sensor is embedded within a Velcro strap that maximize adhesion between PSMS probe and user's fingers. As long as body motion is reduced, as happens in relax and mental stress phases, such criticality does not arise and HRV parameters are very alike, with SDNN percentage difference remaining lower than the $20 \%$. Such problem also explains the increase of $\Delta \%$ in IBI mean and HR mean during the physical stress stage. Expectably, such problem will be solved by integrating the sensor within the ring case (Figure 1) that will maximize the contact area between the finger and the HRV probe, so reducing the quantity of external light reaching the photodiode of the MAXIM 30100.

It is well known that body motion alters tissue optical properties since accelerates blood flowing into local vessels [9], [22]. On such regard, HRV correction obtained by sensor fusion between PSMS and inertial sensors on board the DAPHNE system will be adopted.

GSR results are particularly interesting for \#Startle and Startle Time parameters, which results comparable and characterized by a maximum $\Delta \%$ of $13.9 \%$ in \#Startle and of $19.1 \mathrm{~mm}$ 
$17.2 \%$ in Startle time. The discrepancies observed for the Startle Amp parameter are caused by the different gain featured by amplifiers operating within the SeedGrove sensor and the gold standard. Further improvement of the GSR accuracy can be achieved by implementing a digital potentiometer on board that adapts the resistor value of the GSR probe to the users' skin resistance. In fact, whenever the latter results particularly low $(<150 \mathrm{k} \Omega)$, and the resistor used within the Wheatstone bridge of the GSR probe cannot be tuned, artefacts occur.

\section{CONCLUSIONS}

We presented the preliminary results obtained out of a comparison between a WT system measuring GSR and HRV signals and a gold standard measuring the same parameters, namely the Shimmer3 GSR+. Such solution will be integrated within the DAPHNE system, a WT aimed at the early diagnosis of PD and at enabling remote healthcare services. HRV and GSR measured in low-motion conditions yielded performance particularly comparable to the gold standard chosen. During motion, problems were observed due to light entering into the HRV photodiode. Nevertheless, such problem can be solved by simply integrating into the ring the sensors and optimizing the HRV probe-finger coupling. Importantly, the system proved to successfully follow physiological signal evolutions furnishing reasonable parameters, with the exception of the sole SDNN. For instance, HR mean increases if physical stress is induced or \#Startle decreases upon termination of mental stress. $\rightarrow$ Therefore, implementation of classifiers paradigms for activity recognition can be adopted. However, a much larger set of users has to be used and tests have to be repeated. The possibility to be integrated with inertial sensors, thus exploiting sensor fusion, within an unobtrusive system, such as a ring, tap opens opportunities so as to provide an instrument capable of carry out long-term activity monitoring with high precision. Moreover, Bluetooth connectivity can allow remote AI reasoning so importantly enhancing activity recognition functionalities, the stress detection and promoting active ageing.

\section{REFERENCES}

[1] S. Majumder, T. Mondal, and M. Deen, "Wearable Sensors for Remote Health Monitoring," Sensors, vol. 17, no. 1, p. 130, Jan. 2017.

[2] M. Rinieri, L. Santarelli, L. Fabbri, G. Pastucci, E. Rovini, D. Esposito, S. Pancani, C. Maremmani, F. Vannetti, and F. Cavallo, "Preliminary studies for the evaluation of a novel wearable sensor for biomechanical analysis of upper limbs in Parkinson Disease," Gait Posture, vol. 57, p. 38, Sep. 2017.

[3] E. Rovini, L. Santarelli, D. Esposito, C. Maremmani, and F. Cavallo, "DAPHNE : a novel e-Health system for the diagnosis and the treatment of Parkinson 's Disease," no. July, 2017. P. Kumari, L. Mathew, and P. Syal, "Increasing trend of wearables and multimodal interface for human activity monitoring: A review," Biosensors and Bioelectronics. 2017.

[6]

Physiological Signal Analysis Techniques and Classifiers for Automatic Emotional Evaluation of Audiovisual Contents," Front. Comput. Neurosci., vol. 10, 2016.

6] W. J. Tyler, A. M. Boasso, J. D. Charlesworth, M. A. Marlin, K. Aebersold, L. Aven, D. Z. Wetmore, and S. K. Pal, "Transdermal neuromodulation of noradrenergic activity suppresses psychophysiological and biochemical stress responses in humans," bioRxiv, 2015.

[7] M. van Dooren, J. J. G. G. J. de Vries, and J. H. Janssen, "Emotional sweating across the body: Comparing 16 different skin conductance measurement locations," Physiol. Behav., vol. 106, no. 2, pp. 298-304, 2012.

[8] J. A. Healey and R. W. Picard, "Detecting Stress During RealWorld Driving Tasks Using Physiological Sensors," IEEE Trans. Intell. Transp. Syst., vol. 6, no. 2, pp. 156-166, Jun. 2005.

[9] A. Avakhkisomi, A. Miled, M. Boukadoum, M. Morissette, F. Lellouche, and B. Gosselin, "A novel wireless ring-shaped multisite pulse oximeter," Proc. - IEEE Int. Symp. Circuits Syst., vol. 2016-July, pp. 2451-2454, 2016.

[10] E. Jovanov, A. O’Donnell Lords, D. Raskovic, P. G. Cox, R. Adhami, and F. Andrasik, "Stress monitoring using a distributed wireless intelligent sensor system," IEEE Eng. Med. Biol. Mag., vol. 22, no. 3, pp. 49-55, May 2003.

[11] S. Betti, R. Molino Lova, E. Rovini, G. Acerbi, L. Santarelli, M. Cabiati, S. Del Ry, and F. Cavallo, "Evaluation of an integrated system of wearable physiological sensors for stress monitoring in working environments by using biological markers," IEEE Trans. Biomed. Eng., pp. 1-1, 2017.

[12] J. Schumm, M. Bachlin, C. Setz, B. Arnrich, D. Roggen, and G. Troster, "Effect of movements on the electrodermal response after a startle event," in 2008 Second International Conference on Pervasive Computing Technologies for Healthcare, 2008, pp. 315-318.

[13] E. Rovini, C. Maremmani, and F. Cavallo, "How Wearable Sensors Can Support Parkinson's Disease Diagnosis and Treatment: A Systematic Review," Front. Neurosci., vol. 11, p. 555, Oct. 2017.

[14] F. Cavallo, D. Esposito, E. Rovini, M. Aquilano, M. C. Carrozza, P. Dario, C. Maremmani, and P. Bongioanni, "Preliminary evaluation of SensHand V1 in assessing motor skills performance in Parkinson disease," in IEEE International Conference on Rehabilitation Robotics, 2013.

[15] F. Cavallo, C. Maremmani, D. Esposito, E. Rovini, P. Dario, and M. C. Carrozza, "Method and related apparatus for monitoring biomechanical performances of human limbs," WO2014108883 A1. PCT/IB2014/058265, 2013.

[16] J. T. RamShur, "HRVAS: Heart Rate Variability Analysis Software." [Online]. Available:

ftp://mirror.ac.za/sourceforge/h/project/project/hr/hrvas/Documen ts/HRVAS_user_manual.pdf. [Accessed: 01-Feb-2018].

[17] W. Boucsein, Electrodermal activity. Springer Science+Business Media, LLC, 2012.

[18] M. Benedek and C. Kaernbach, "A continuous measure of phasic electrodermal activity," J. Neurosci. Methods, vol. 190, no. 1, pp. 80-91, 2010.

[19] P. F. Lovibond, "Tonic and Phasic Electrodermal Measures of Human Aversive Conditioning with Long Duration Stimuli," Psychophysiology, vol. 29, no. 6, pp. 621-632, Jan. 2007.

[20] C. Setz, B. Arnrich, J. Schumm, R. La Marca, G. Troster, and U. Ehlert, "Discriminating Stress From Cognitive Load Using a Wearable EDA Device," IEEE Trans. Inf. Technol. Biomed., vol. 14, no. 2, pp. 410-417, Mar. 2010.

[21] R. Hoffman and M. Al'Absi, "The effect of acute stress on subsequent neuropsychological test performance (2003)," Arch. Clin. Neuropsychol., vol. 19, no. 4, pp. 497-506, Jun. 2004.

[22] A. N. A N Bashkatov, E. A. Genina, V. I. Kochubey, and V. V Tuchin, "Optical properties of human skin, subcutaneous and mucous tissues in the wavelength range from 400 to $2000 \mathrm{~nm}$," $J$. Phys. D. Appl. Phys., vol. 38, no. 15, pp. 2543-2555, Aug. 2005. 